\title{
Discrete subpulmonic membrane in transposition of great arteries with an intact ventricular septum
}

\author{
Trushar Gajjar, Nageswar Rao, Neelam Desai \\ Received: November 23, 2014 Accepted: January 06, 2015 Published online: February 10, 2015
}

The left ventricular outflow tract obstruction is rare in transposition of the great arteries with an intact ventricular septum. The obstruction may be dynamic or anatomic. Herein, we present a four-year-old boy who was diagnosed with dextro-transposition of the great arteries, patent ductus arteriosus with an intact ventricular septum, and left ventricular outflow tract obstruction due to a discrete subpulmonic membrane.

Keywords: Left ventricular outflow tract obstruction; subpulmonic membrane; transposition of great arteries.

The left ventricular outflow tract (LVOT) obstruction is rare in transposition of the great arteries with an intact ventricular septum (TGA/IVS). Only in $0.7 \%$ of cases this combination is reported. ${ }^{[1]}$ The obstruction may be dynamic or fixed. ${ }^{[1]}$ Herein, we present a case report of this rare anomaly with various treatment options.

\section{CASE REPORT}

A four-year-old child presented with cyanosis since birth. On physical examination, uniform central cyanosis and clubbing were present. The heart rate was $90 / \mathrm{min}$ on a regular basis and blood pressure was $120 / 70 \mathrm{mmHg}$. Electrocardiogram showed sinus rhythm with right axis deviation. Chest X-ray revealed situs solitus, left ventricular type of apex, biatrial enlargement and pulmonary plethora. Two dimensional echocardiography showed (Figure 1a) dextro-transposition of the great arteries (d-TGA), an intact interventricular septum, subpulmonic membrane (subcostal view), LVOT gradient of $43 / 26 \mathrm{mmHg}$, an aneurysm of interatrial septum, patent ductus arteriosus (PDA), morphologically regressed left ventricle and good ventricular function. Left ventriculogram showed a discrete subpulmonic membrane with $46 \mathrm{mmHg}$ gradient (Figure 1b), an intact ventricular septum with good-size main and branch pulmonary arteries and a small PDA. In view of morphologically regressed left ventricle and significant gradient across the LVOT, we decided to perform Sennings operation with excision of subpulmonic membrane.
The patient was taken for surgery and a conventional median sternotomy approach was used. Preoperative findings revealed d-TGA, and the aorta to the right and anterior to the pulmonary artery. The coronary arteries were normal for d-TGA with sinus-1 giving the left coronary artery and the sinus- 2 giving the right coronary artery. Both the atria were of good size with an absent atrial isomerism. There was a large secundum type of atrial septal defect and a complete diaphragm like subpulmonic membrane with a central opening (Figure 2a). Pulmonary valve was tricuspid with thickened leaflets and a small PDA. The subpulmonic membrane was excised, the PDA was ligated and a physiological correction was done by a modified Sennings operation. The weaning from cardiopulmonary bypass was done in a usual way.

The postoperative course was uneventful and an immediate postoperative echocardiography revealed nonobstructed systemic and pulmonary venous return, no residual shunt, mild tricuspid regurgitation, trivial pulmonary regurgitation; gradient across the LVOT was $6 \mathrm{mmHg}$ with mild right ventricular dysfunction and a normal left ventricular function. A three month and two year follow-up showed asymptomatic patient and echocardiography revealed normal systemic and

Department of Cardiothoracic and Vascular Surgery, Sri Sathya Sai Institute of Highar Medical Sciences, Prasanthigram, Andhra Pradesh, India

Corresponding author: Trushar Gajjar, M.D. Department of Cardiothoracic and Vascular Surgery, Sri SathyaSai Institute of Higher Medical Sciences, Prashanthigram 515134, District Anantapur, Andhra Pradesh, India.

Tel: +91 9700355411 e-mail: trushargajjar@gmail.com 

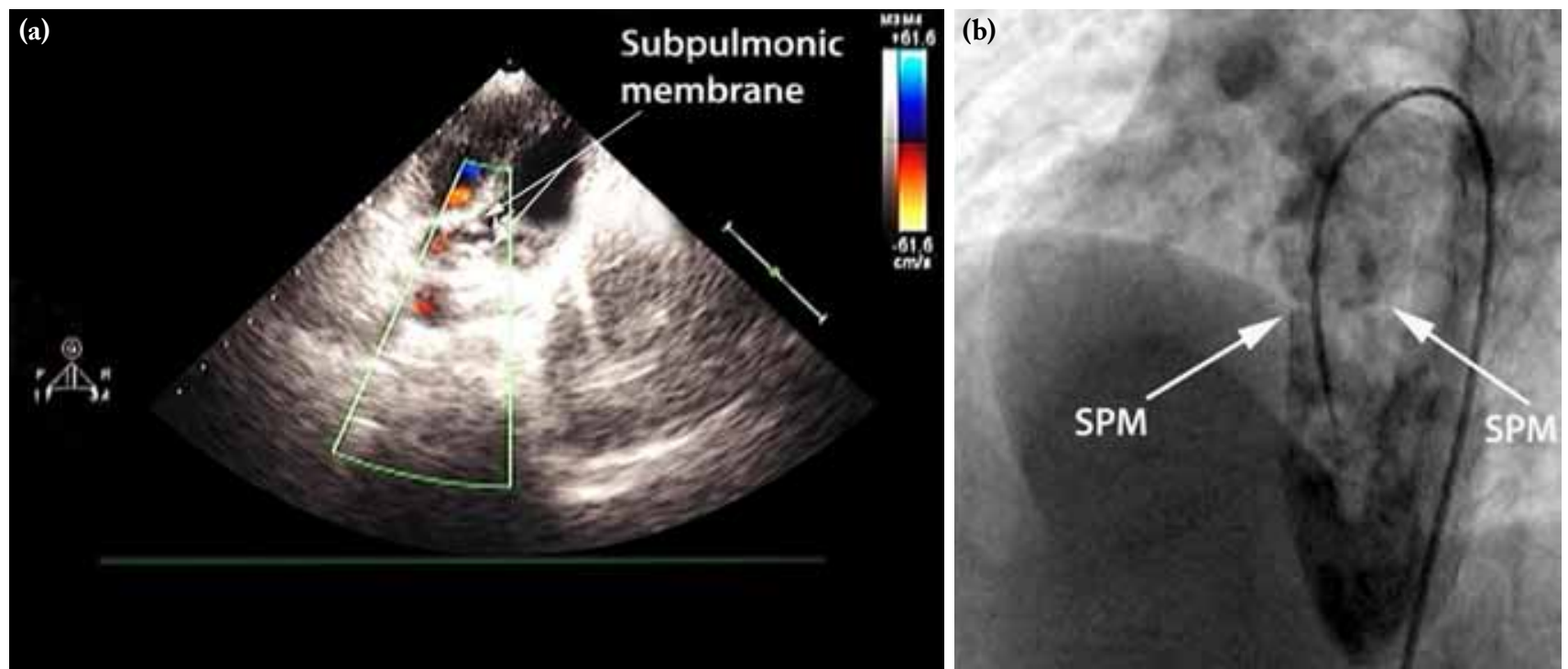

Figure 1. (a) Transthoracic echocardiogram in a subcostal view showing a discrete subpulmonic membrane. (b) A left ventriculogram through the femoral artery to descending aorta to patent ductus arteriosus to main pulmonary artery to left ventricle showing intact interventricular septum and discrete subpulmonic membrane. SPM: Subpulmonic membrane.

pulmonary venous drainage, insignificant gradient across the LVOT and a good ventricular function.

\section{DISCUSSION}

Left ventricular outflow tract obstruction is well-known in overall $30-35 \%$ of patients in association with TGA. ${ }^{[1]}$ However, only $0.7 \%$ of patients with TGA and intact ventricular septum develop a critical LVOT obstruction. ${ }^{[1]}$ Very few cases of discrete subpulmonic membrane have been reported in $\mathrm{d}$-TGA/IVS in the literature. ${ }^{[1]}$ The obstruction may be dynamic or anatomic with anatomic causes ranging from the subvalvar fibrous ridge to fibrous tags, hypertrophied bands and valvar stenosis. ${ }^{[1]}$ A discrete subpulmonic membrane causing LVOT obstruction in patients with d-TGA is rare, although it has been reported with doubly committed ventricular septal defect in association with subpulmonary fibrous ridge. ${ }^{[2-5]}$ Ozkutlu et al. ${ }^{[2]}$ reported a discrete subaortic and subpulmonic stenosis with doubly committed ventricular septal defect in a series of nine patients. Raff et al. $^{[3]}$ reported a subpulmonic membrane in association with a ventricular septal defect and aortic insufficiency. Duggal et al. ${ }^{[4]}$ showed a subpulmonic membrane in association with a supracristal ventricular septal defect. Tomar et al. ${ }^{[5]}$ recommend a careful echocardiography of all similar presentations which may enable us to learn more of this rare lesion. The pathophysiology of LVOT obstruction in TGA/IVS shows that a dynamic type of obstruction is common. ${ }^{[1]}$ A fixed type of obstruction is very rare and of two

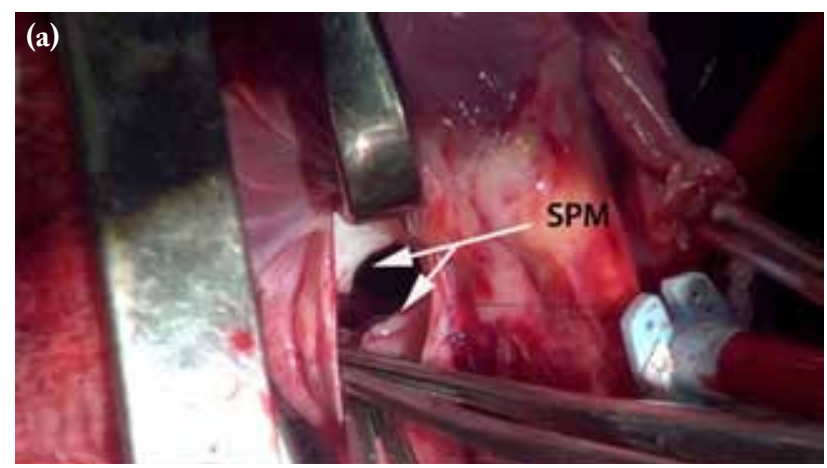

(b)

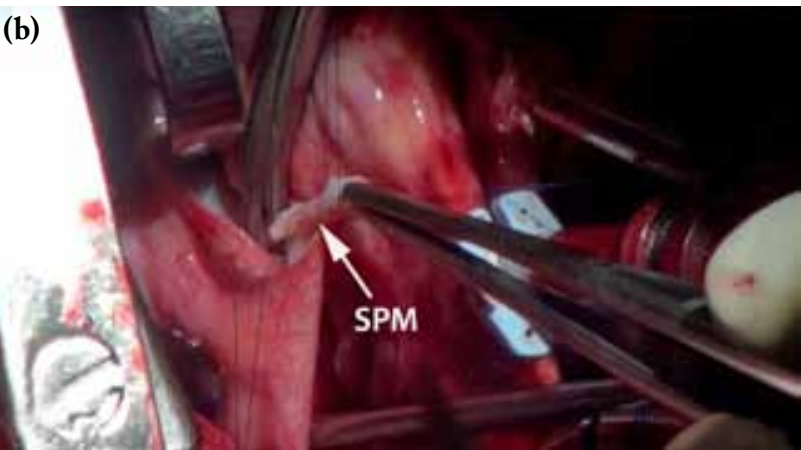

Figure 2. Intraoperative pictures taken from the head end of the table showing, (a) a complete fibrous ring just below the pulmonary valve, (b) excised subpulmonic ring. SPM: Subpulmonic membrane. 
types, discrete or tunnel type. ${ }^{[1]}$ The discrete variety initially appears as a patch of endocardial thickening on the septal bulge, but later may evolve into sharp fibrous ridge or a discrete membrane which is analogous to the subaortic membrane. ${ }^{[1]}$ The less common type of fixed subpulmonary obstruction is caused by a tunnel like subpulmonary fibromuscular ridge that extends across the outflow tract. ${ }^{[1]} \mathrm{A}$ careful transthoracic echocardiography in subcostal and apical five-chamber view can be useful in the diagnosis of this rare anomaly. Although percutaneous dilatation of subpulmonic membrane has been reported in the literature,${ }^{[5]}$ the surgical excision is the treatment of choice along with the definitive surgical procedure. If this patient would have presented early with normal, non-regressed left ventricle Arterial switch would be the preferred operation. Unfortunately, our patient presented late with regressed left ventricle. In case of a significant and borderline left ventricle, the left ventricle can be trained in the late arterial switch operation. On the other hand, in case of a significantly preferably gradient less than $40 \mathrm{mmHg}$ and morphologically regressed membrane excision of the left ventricle can be avoided to keep slightly higher left ventricular pressure which in long-term helps to improved right ventricular function. In case that corrected transposition (Corrected TGA) subpulmonic membrane can produce pulmonary insufficiency like subaortic membrane, but with lower pulmonary artery pressure, the chances of pulmonary insufficiency are rare. In the absence of a non-significant obstruction, probably gradient less than $40 \mathrm{mmHg}$ without associated with cardiac anomaly requiring surgical correction, the subpulmonic membrane can be left alone, however, there is a possibility of progression of gradient later in life due to the development of fibrosis secondary to turbulence. However, if the gradient is more than $40 \mathrm{mmHg}$ without associated with cardiac anomaly or associated cardiac anomaly is present in spite of gradient less than $40 \mathrm{mmHg}$ in, corrected TGA resection of subpulmonic membrane is advisable.

In conclusion, a discrete subpulmonic membrane in d-TGA with IVS is a rare anomaly, careful transthoracic echocardiography is able to diagnose this rare entity and surgical excision is the treatment of choice along with the definitive surgical procedure.

\section{Declaration of conflicting interests}

The authors declared no conflicts of interest with respect to the authorship and/or publication of this article.

\section{Funding}

The authors received no financial support for the research and/or authorship of this article.

\section{REFERENCES}

1. Wernovsky G. Transposition of the great arteries. In: Allen HD, Driscoll DJ, Shaddy RE, Feltes TF, editors. Moss and Adams' Heart Disease in Infants, Children, and Adolescents: Including the Fetus and Young Adults. 7th ed. New York: Lippincott Williams \& Wilkins; 2008; 1045-47.

2. Ozkutlu S, Saraçlar M, Alehan D, Yurdakul Y, Firat P, Tokel K. Subpulmonary and subaortic ridges in doubly committed subarterial ventricular septal defect: an echocardiographic study. Eur Heart J 1996;17:935-9.

3. Raff GW, Gaynor JW, Weinberg PM, Spray TL, Gleason M. Membranous subpulmonic stenosis associated with ventricular septal defect and aortic insufficiency. J Am Soc Echocardiogr 2000;13:58-60.

4. Duggal B, Bajaj M, Bansal NO. Subpulmonic membrane associated with a supracristal VSD: a rare cause of right ventricular outflow obstruction. Echocardiography 2011;28:E89-90.

5. TomarM,RadhakrishnanS, Sharma R. Isolated subpulmonary membrane causing critical neonatal pulmonary stenosis with concordant atrioventricular and ventriculoarterial connections. Images Paediatr Cardiol 2009;11:5-9. 